\title{
Potential Protective Effect of Achillea fragrantissima against Adriamycin-Induced Cardiotoxicity in Rats via an Antioxidant and Anti-Inflammatory Pathway
}

\author{
Maha A. Hijazi (D), Hanan A. Jambi, Buthaina M. Aljehany, and Maha A. Althaiban \\ Food and Nutrition Department, Faculty of Home Economics, King Abdulaziz University, Jeddah, Saudi Arabia \\ Correspondence should be addressed to Maha A. Hijazi; maha.hej@gmail.com
}

Received 18 January 2019; Revised 24 April 2019; Accepted 7 May 2019; Published 17 June 2019

Academic Editor: Kota V. Ramana

Copyright (c) 2019 Maha A. Hijazi et al. This is an open access article distributed under the Creative Commons Attribution License, which permits unrestricted use, distribution, and reproduction in any medium, provided the original work is properly cited.

\begin{abstract}
Adriamycin (Adr) is a cytotoxic anthracycline agent that is utilized to manage many types of tumors, but its clinical use is undesirable due to severe cardiotoxicity. The present study aimed to investigate the cardioprotective effect of Achillea fragrantissima (A. fragrantissima) against Adr-induced cardiotoxicity through the antioxidant and anti-inflammatory metabolic pathways. A single dose of Adr was injected in rats to induce cardiotoxicity. Rats are divided into 5 groups, control, A. fragrantissima 800, Adr, A. fragrantissima $400+$ Adr, and A. fragrantissima $800+$ Adr. $72 \mathrm{~h}$ after Adr administration, electrocardiographic (ECG) study was performed for all rats. Serum and hearts were then collected for biochemical and histopathological studies. A. fragrantissima ameliorated Adr-induced ST-segment elevation. It reduced Adr-induced elevation in lactate dehydrogenase (LDH), creatine kinase$\mathrm{MB}$ (CK-MB), thiobarbituric acid reactive substance (TBARS), tumor necrosis factor-alpha (TNF- $\alpha$ ), interleukin-1 beta (IL-1 $\beta$ ), and IL-6. It also protected against Adr-induced histopathological changes. Pretreatment with the extract increased heart tissue contents of glutathione peroxidase (GSH-PX) and reduced glutathione (GSH). Phytochemical analysis of the extract revealed that it is rich in phenolic and flavonoid active constituents. The results of this study revealed that A. fragrantissima extract ameliorates Adrinduced cardiotoxicity via an antioxidant and anti-inflammatory mechanisms. Further studies are warranted in order to recognize the precise active constituents of this natural extract which are responsible for the antioxidant and anti-inflammatory actions.
\end{abstract}

\section{Introduction}

Adriamycin (Adr) is a cytotoxic anthracycline antibiotic that is used to treat a wide variety of cancers including, leukaemia and lymphoma, besides breast, lung, and other solid cancers. However, its clinical application is restricted due to its dangerous cardiotoxicity that may be aggravated to heart failure [1-3]. The underlying mechanism of Adrinduced acute cardiotoxicity is based on the production of free radicals and reactive oxygen species (ROS) that damage the cell membrane lipids and caused the liberation of lipid peroxides products $[4,5]$. Moreover, there is growing evidence that Adr also elicits inflammatory effects in the vasculature and the myocardium that subsequently induces the production of several proinflammatory mediators such as tumor necrosis factor-alpha (TNF- $\alpha$ ) [6]. Interleukin-1 beta $(\mathrm{IL}-1 \beta)$ is an initiator cytokine that exhibits a great function as a regulator of the immunoinflammatory pathway. IL- $1 \beta$ has been disclosed to contribute to the Adr-induced rises in the levels of IL- 6 and perform a very distinct role in Adr-induced cardiotoxicity [7]. Research has found that cardiac toxicity of Adr is also associated with an increase in IL- $1 \beta$ production. Il$1 \mathrm{~B}$ causes cardiomyocytes apoptosis by increasing the calcium storage of the heart muscle cells [8].

Recently, many studies documented that natural products rich in antioxidants have an effective role in the prevention of cardiac toxicity of Adr [9]. The preventive mechanism for natural remedies against Adr-induced cardiotoxicity is still unclear. However, research has shown that the mechanism may be limited to the elimination of ROS or prevention against the harmful cardiovascular effects of the metabolic syndrome [10-12]. A. fragrantissima is one of the desert herbs belonging to the family Asteraceae. Historically, the herb is used in the Arab region as an alternative treatment for 
diabetes, gastrointestinal diseases, and respiratory diseases [13-16]. A. fragrantissima contains a high percentage of phenolic and flavonoid active constituents such as achillolide A, swertisin $2^{\prime \prime}$-arabinosideafroside, cirsimaritin, chrysoplenol, cirsiliol, eupatilin-7-methyl ether, and isovitexin $4^{\prime}$ - methyl ether [17-19]. Sesquiterpene lactones are a group of active substances, which have shown a great biological efficacy as anti-inflammatory, antioxidant, and neuroprotective compounds [20-23]. Achillolide A, a sesquiterpene lactone, is one of the most effective substances separated from many of the Asteraceae family, which has shown a strong antiinflammatory effect and sweeping ROS and also prevents the formation of nitric oxide (NO) [18].

Since oxidative stress and inflammation have become accepted as a suitable target for early therapeutic intervention in Adr-induced cardiotoxicity, the present study addressed the cardioprotective effect of A. fragrantissima against Adrinduced cardiotoxicity through the antioxidant and antiinflammatory metabolic pathways.

\section{Methods}

2.1. Drugs. Drugs included are Adr (Adriamycin ${ }^{\circledR}$ ) $50 \mathrm{mg} / 25 \mathrm{ml}$, EBEWE Pharma, Austria, Urethane (Sigma Aldrich Inc., USA).

2.2. Collection of A. fragrantissima. The plant was collected from Al-Jawf in Northern Saudi Arabia. The taxonomic identification of the plant was confirmed by botanists in Pharmaceutical and Phytochemistry Department, Faculty of Pharmacy, KAU, Jeddah, Saudi Arabia.

2.3. Preparation of Plant Extract. The aerial parts of $A$. fragrantissima were dried at $40^{\circ} \mathrm{C}$ and grinded. $500 \mathrm{~g}$ of $A$. fragrantissima powder was then extracted with methanol and water mixture (80\%). The final collected extract was filtrated and concentrated to dryness under reduce pressure at $<$ $35^{\circ} \mathrm{C}$ using a rotary evaporator (Rota vapor R-215, Bütchi, Switzerland). Finally, the dried extract was transferred to a Gamma 2-20 freeze dryer (Christ, Osterode i. H., Germany) for $48 \mathrm{~h}$, at $-20^{\circ} \mathrm{C}$ to yield a solid extract (100 g yield $4.27 \mathrm{~g}$ ), and then stored at $4^{\circ} \mathrm{C}$ until further use. $100 \mathrm{mg}$ of dried $A$. fragrantissima extract was dissolved in $200 \mathrm{ml}$ distilled water to yield a solution with a final concentration of $0.5 \mathrm{mg} / \mathrm{ml}$, centrifuged to remove any undissolved component [24].

2.4. Phytochemical Analysis. The phytochemical screening of A. fragrantissima extract has been performed to find the presence of the major chemical constituents, including alkaloids, flavonoids, glycosides, saponins, tannins, resins, and triterpenoids using standard procedures of analysis [25].

2.5. Experimental Design. In this study, 50 adult male Wister rats (180-200 g body weight) were divided into 5 groups $(\mathrm{n}=$ 10). The rats were obtained from King Fahd medical research center, KAU, Jeddah, Saudi Arabia. They handled according to the roles and rights of animal research, KAU. The rats housed at $22 \pm 3^{\circ} \mathrm{C}$ and maintained free axis to food and water.
The study groups were as follows: Group (I) (control), the rats injected with normal saline; Group (II) (A. fragrantissima $800)$, the rats orally injected with $A$. fragrantissima extract at a dose of $800 \mathrm{mg} / \mathrm{kg} /$ day; Group (III) (Adr), the rats injected i.p. with Adr (10 mg/kg) [26]; Group (IV) (A. fragrantissima 400 + Adr), the rats orally injected with $A$. fragrantissima extract at a dose of $400 \mathrm{mg} / \mathrm{kg} /$ day for 2 weeks [27] and then injected with Adr (10 mg/kg); Group (V) (A. fragrantissima $800+$ Adr), the rats orally injected with $A$. fragrantissima extract at a dose of $800 \mathrm{mg} / \mathrm{kg} /$ day for 2 weeks and then injected with Adr (10 mg/kg).

2.6. Electrocardiography (ECG) Assessment. $72 \mathrm{~h}$ after $\mathrm{Adr}$ injection, the PowerLab system (ADI Instruments) connected to a PC running LabChart professional software (version 7.3) containing an ECG module was used to conduct the standard limb lead II of the surface ECG for each rat separately [28].

2.7. Samples Collection. $72 \mathrm{~h}$ after Adr injection, rats were anesthetized with ether, blood samples were collected, and serum was separated and kept frozen at $-80^{\circ} \mathrm{C}$ for the biochemical determination of the cardiac enzymes and inflammatory cytokines (IL- $1 \beta$, TNF- $\alpha$, and IL-6). The rats were then decapitated, and the hearts were collected and maintained either frozen at $-80^{\circ} \mathrm{C}$ for the ELIZA determination of thiobabituric acid reactive substances (TBARS), glutathione peroxidase (GSH-PX), and reduced glutathione $(\mathrm{GSH})$ or in $10 \%$ buffered formalin solution for the histopathological study.

2.8. Determination of Serum Creatine Kinase-MB (CK-MB) and Lactate Dehydrogenase (LDH). Serum CK-MB and LDH activity were determined using ELISA kits obtained from MyBiosource, San Diego, California, USA, according to the manufacture instructions.

2.9. Preparation of Heart Tissues Homogenate. The heart tissues were homogenized in PBS (1:9) using a Teflon pestle (Ultra-Turrax, IKA: T25 digital, Germany) and centrifuged at $12000 \mathrm{~g}$ for $15 \mathrm{~min}$ at $4^{\circ} \mathrm{C}$ (Centurion, K280 R, UK). The supernatants were used for the estimation of TBARS, GSH$\mathrm{PX}$, and GSH.

2.10. Determination of Heart Tissue TBARS, GSH-PX, and GSH Concentration. TBARS, GSH-PX, and GSH were determined using ELISA kits obtained from MyBiosource, San Diego, California, USA, according to the manufacture instructions.

2.11. Determination of Serum TNF- $\alpha$, IL-1 $\beta$, and IL-6 Concentration. TNF- $\alpha$, IL- $1 \beta$, and IL- 6 were determined using ELISA kits obtained from MyBiosource, San Diego, California, USA, according to the manufacture instructions.

2.12. Histopathological Examination. The formaldehyde fixed hearts were paraffin-embedded, cut into sections, and then 
stained with Hematoxylin-Eosin ( $\mathrm{H} \& \mathrm{E})$. The slides were then examined microscopically.

2.13. Statistical Analysis. Results were presented as mean $\pm \mathrm{SE}$. The means were compared by one-way analysis of variance (ANOVA), followed by Tukey's HSD, to determine the statistical significance of the difference using SPSS version 22. $\mathrm{P} \leq 0.05$ indicate significance difference.

\section{Results}

3.1. Phytochemical Analysis of A. fragrantissima Extract. Phytochemical analysis of $A$. fragrantissima extract indicated that it contains adequate amounts of flavonoids, phenolics, glycosides, lignans, and terpenes (Table 1).

\subsection{Effects of A. fragrantissima on Adr-Induced Changes in} ECG Tracing Pattern and Parameters. The ECG of the control and $A$. fragrantissima 800 groups presented a normal-pattern (Figures 1(a) and 1(b)). Injection of rats with $\mathrm{Adr}(10 \mathrm{mg} / \mathrm{kg})$ induced remarkable ST-segment elevation (Figure 1(c)). Pretreatments of rats with $A$. fragrantissima extract at both 400 and $800 \mathrm{mg} / \mathrm{kg}$ ameliorated Adr-induced ST-segment elevation (Figures $1(\mathrm{~d})$ and $1(\mathrm{e})$ ). In addition, injection of rats with $\operatorname{Adr}(10 \mathrm{mg} / \mathrm{kg})$ significantly $(\mathrm{p} \leq 0.05)$ increased heart rate and QT interval compared to the control group (Table 2). Pretreatments of rats with A. fragrantissima extract at both 400 and $800 \mathrm{mg} / \mathrm{kg}$ had no effect on Adr-induced increase in both heart rate and QT interval (Table 2).

\subsection{Effects of A. fragrantissima on Serum CK-MB and $L D H$} Measured in Adr-Induced Cardiotoxicity in Rats. Injection of rats with $\mathrm{Adr}(10 \mathrm{mg} / \mathrm{kg})$ significantly elevated ( $\mathrm{p} \leq 0.05)$ both serum CK-MB and LDH levels compared to the control group. Pretreatments of rats with A. fragrantissima extract at both 400 and $800 \mathrm{mg} / \mathrm{kg}$ significantly reduced $(\mathrm{p} \leq 0.05)$ both serum CK-MB and LDH levels compared to the Adr group. However, rats pretreated with $800 \mathrm{mg} / \mathrm{kg}$ A. fragrantissima showed significantly lower $(\mathrm{p} \leq 0.05)$ serum $\mathrm{CK}-\mathrm{MB}$ and $\mathrm{LDH}$ levels compared to $400 \mathrm{mg} / \mathrm{kg}$ A. fragrantissima $+\mathrm{Adr}$ group (Table 3 ).

\subsection{Effects of A. fragrantissima on Heart Tissue Concentration} of TBARS, GSH-PX, and GSH Measured in Adr-Induced Cardiotoxicity in Rat. Injection of rats with $\mathrm{Adr}(10 \mathrm{mg} / \mathrm{kg})$ significantly elevated ( $\mathrm{p} \leq 0.05$ ) heart tissue TBARS concentration compared to the control group. Pretreatments of rats with $A$. fragrantissima extract at both 400 and $800 \mathrm{mg} / \mathrm{kg}$ significantly reduced ( $\mathrm{p} \leq 0.05$ ) heart tissue TBARS concentration compared to the Adr group. However, rats pretreated with $800 \mathrm{mg} / \mathrm{kg}$ A. fragrantissima showed significantly lower ( $\mathrm{p} \leq 0.05$ ) heart tissue TBARS concentration compared to $400 \mathrm{mg} / \mathrm{kg}$ A. fragrantissima + Adr group (Table 4).

Injection of rats with $\mathrm{Adr}(10 \mathrm{mg} / \mathrm{kg})$ significantly reduced ( $\mathrm{p} \leq 0.05$ ) both heart tissue GSH-PX and GSH concentration compared to the control group. Pretreatments of rats with $A$. fragrantissima extract at both 400 and $800 \mathrm{mg} / \mathrm{kg}$ significantly elevated $(\mathrm{p} \leq 0.05)$ both heart tissue GSH-PX and GSH
TABLE 1: Phytochemical analysis of $A$. fragrantissima extract.

\begin{tabular}{lc}
\hline Phytochemical compounds & Inference \\
\hline Flavonoids & +++ \\
Phenolic & +++ \\
Glycosides & +++ \\
Terpenoids & ++ \\
Tannins & ++ \\
Alkaloids & ++ \\
Lignans & +++ \\
Steroids & ++ \\
Terpenes & +++ \\
Saponins & + \\
Anthraquinone & + \\
Triterpenes & - \\
Resins & - \\
\hline
\end{tabular}

The intensity of compounds: - absence, + minute, ++ small, and +++ large.

concentration compared to the Adr group. However, rats pretreated with $800 \mathrm{mg} / \mathrm{kg} \mathrm{A}$. fragrantissima showed significantly higher $(\mathrm{p} \leq 0.05)$ both heart tissue GSH-PX and GSH concentration compared to $400 \mathrm{mg} / \mathrm{kg}$ A. fragrantissima + Adr group (Table 4).

3.5. Effects of A. fragrantissima on Serum TNF- $\alpha$, IL-1 $\beta$, and IL-6 Measured in Adr-Induced Cardiotoxicity in Rats. Injection of rats with $\mathrm{Adr}(10 \mathrm{mg} / \mathrm{kg})$ significantly elevated $(\mathrm{p}$ $\leq 0.05$ ) serum TNF- $\alpha$, IL- $1 \beta$, and IL- 6 levels compared to the control group. Pretreatments of rats with A. fragrantissima extract at both 400 and $800 \mathrm{mg} / \mathrm{kg}$ significantly reduced ( $\mathrm{p}$ $\leq 0.05$ ) serum TNF- $\alpha$, IL- $1 \beta$, and IL- 6 levels compared to the Adr group. However, rats pretreated with $800 \mathrm{mg} / \mathrm{kg} A$. fragrantissima showed significantly lower $(\mathrm{p} \leq 0.05)$ serum TNF- $\alpha$, IL- $1 \beta$, and IL-6 levels compared to $400 \mathrm{mg} / \mathrm{kg} A$. fragrantissima + Adr group (Figures 2, 3, and 4).

3.6. Effects of A. fragrantissima on the Heart Tissue Histopathological Changes Detected by $H$ \& E Staining in Adr-Induced Cardiotoxicity in Rat. Figures 5(a) and 6(a) showed control heart with normal histology. Figures 5(b) and 6(b) showed A. fragrantissima 800 heart with nearly normal histology. Injection of $\mathrm{Adr}(10 \mathrm{mg} / \mathrm{kg})$ resulted in focal areas of necrosis, perivascular edema around a coronary blood vessel, marked disorganization of the thin degenerated fibers (Figures 5(c) and $5(\mathrm{~d})$ ). The high-power examination revealed a marked focal aggregation of inflammatory mononuclear cells in Adr-treated rats (Figure 6(c)). The heart tissue of the rats treated with $A$. fragrantissima extract at $400 \mathrm{mg} / \mathrm{kg}$ before Adr showed focal areas of necrosis (Figures 5(e) and 6(d)). On the other hand, pretreatment of Adr injected rats with $A$. fragrantissima extract at $800 \mathrm{mg} / \mathrm{kg}$ resulted in nearly complete protection from Adr-induced histopathological changes (Figures 5(f) and 6(e)). 


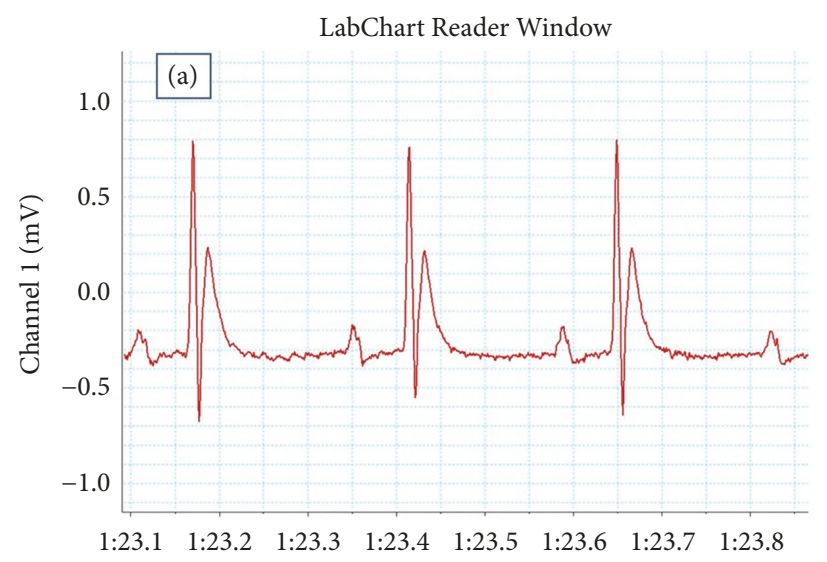

(a)

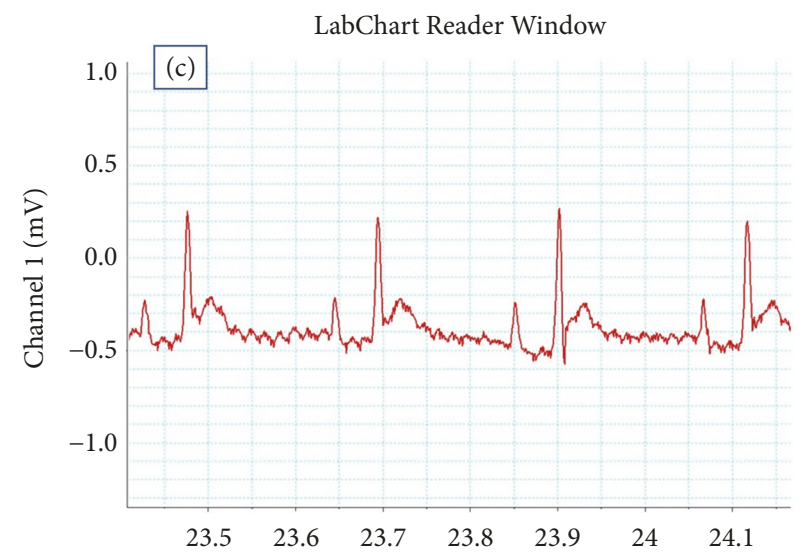

(c)

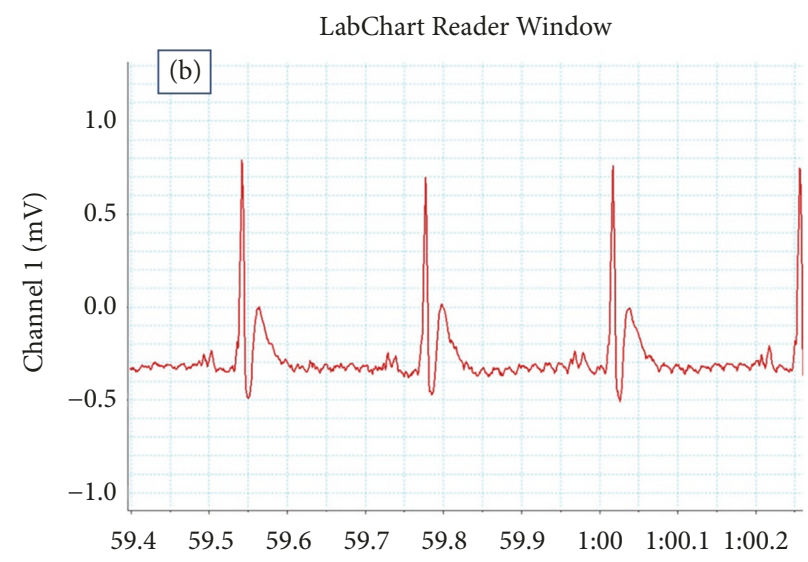

(b)

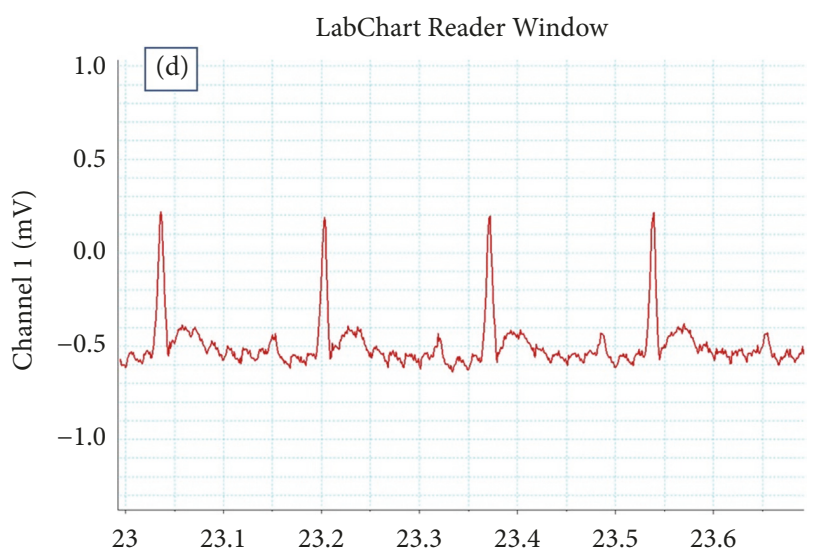

(d)

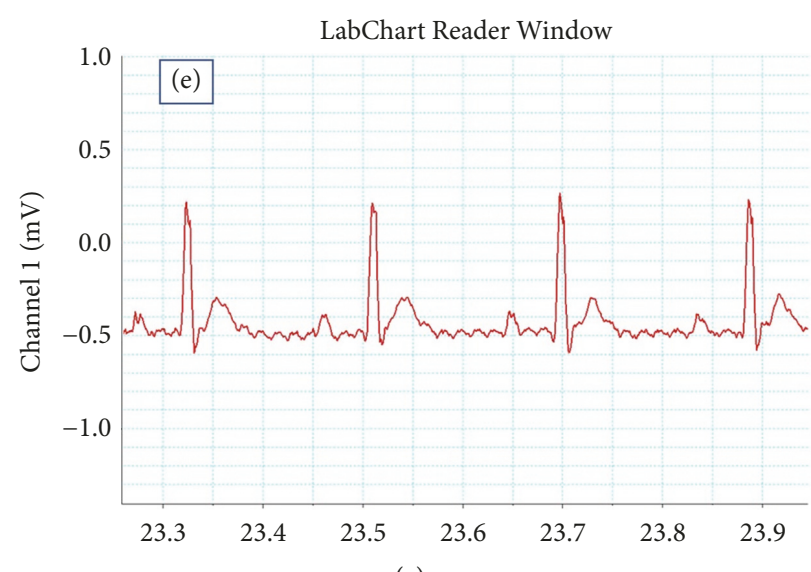

(e)

FIGURE 1: Effects of A. fragrantissima on Adr-induced changes in ECG tracing pattern. (a) Control group, (b) A. fragrantissima 800 group, (c) Adr group, (d) A. fragrantissima $400+$ Adr group, and (e) A. fragrantissima $800+$ Adr group.

\section{Discussion}

Adriamycin is one of the most effective chemotherapeutic agents which is widely used in the treatment of many tumors. The clinical uses of Adr are hampered by many associated toxicities, such as cardiac, renal, and pulmonary toxicity [29]. Adr administration may cause acute cardiac toxicity, which ranges from both ventricular and atrial arrhythmia to congestive heart failure [30,31]. This study aimed to investigate the possible protective effect of $A$. fragrantissima extract against Adr-induced acute cardiotoxicity in rats.

The results of this study revealed that pretreatment of Adr injected rats with $A$. fragrantissima extract protected the rats against Adr-induced cardiotoxicity as manifested 
TABLE 2: Effects of A. fragrantissima on control and Adr-induced changes in EGG parameters.

\begin{tabular}{lcc}
\hline Experimental Groups & $\begin{array}{c}\text { Heart Rate } \\
\text { (beat/min) }\end{array}$ & $\begin{array}{c}\text { QT interval } \\
(\mathrm{s})\end{array}$ \\
\hline Control & $251 \pm 13$ & $0.052 \pm 0.003$ \\
A. fragrantissima 800 & $255 \pm 15$ & $0.05 \pm 0.001$ \\
Adr & $319 \pm 19^{\mathrm{a}}$ & $0.071 \pm 0.004^{\mathrm{a}}$ \\
A. fragrantissima $400+\mathrm{Adr}$ & $328 \pm 10$ & $0.069 \pm 0.005$ \\
A. fragrantissima $800+$ Adr & $316 \pm 12$ & $0.071 \pm 0.002$ \\
\hline
\end{tabular}

Data are represented as mean $\pm \mathrm{SE}(\mathrm{n}=10)$. ${ }^{\mathrm{a}}$ Significant versus control group. $\mathrm{p} \leq 0.05$.

TABLE 3: Effects of A. fragrantissima on serum CK-MB and LDH measured in control and Adr-induced cardiotoxic rats.

\begin{tabular}{lcc}
\hline Experimental Groups & CK-MB & $\begin{array}{c}\text { LDH } \\
(\mathrm{U} / \mathrm{L})\end{array}$ \\
\hline Control & $257.27 \pm 10.87$ & $249.29 \pm 11.74$ \\
A. fragrantissima 800 & $253.4 \pm 9.95$ & $247.96 \pm 12.10$ \\
Adr & $438.52 \pm 15.87^{\mathrm{a}}$ & $529.89 \pm 14.72^{\mathrm{a}}$ \\
A. fragrantissima $400+\mathrm{Adr}$ & $306.01 \pm 10.75^{\mathrm{b}}$ & $316.28 \pm 14.91^{\mathrm{b}}$ \\
A. fragrantissima $800+\mathrm{Adr}$ & $276.40 \pm 8.09^{\mathrm{b}, \mathrm{c}}$ & $271.78 \pm 8.10^{\mathrm{b}, \mathrm{c}}$ \\
\hline
\end{tabular}

Data are represented as mean \pm SE $(n=10)$. ${ }^{a}$ Significant versus control group, ${ }^{\mathrm{b}}$ significant versus Adr group, and ${ }^{\mathrm{C}}$ significant between low and high dose group. $\mathrm{p} \leq 0.05$.

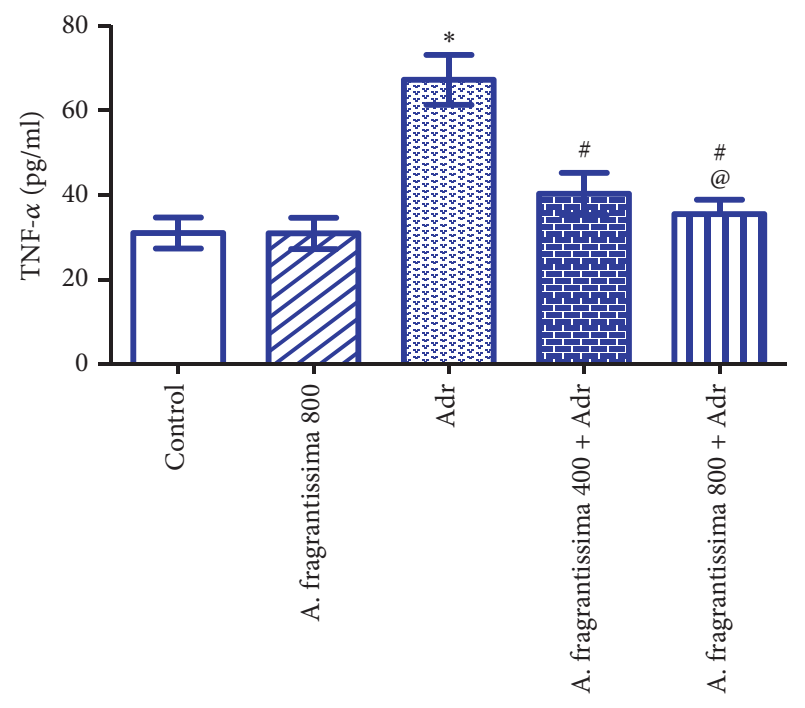

FIGURE 2: Effects of A. fragrantissima on serum concentration of TNF- $\alpha$ measured in control and Adr-induced cardiotoxic rats. Data are represented as mean \pm SE $(n=10)$. ${ }^{*}$ Significant versus control group, " significant versus Adr group, and ${ }^{\circledR}$ significant between low and high dose group. $\mathrm{p} \leq 0.05$.

by the decreased cardiac enzymes, LDH and CK-MB. In addition, the histopathological study of the heart muscle revealed that $A$. fragrantissima extract improved Adr-induced histopathological and ultrastructural damage.

In this study, we found that Adr moderately prolonged QT interval, increased heart rate, and provoked ST-segment

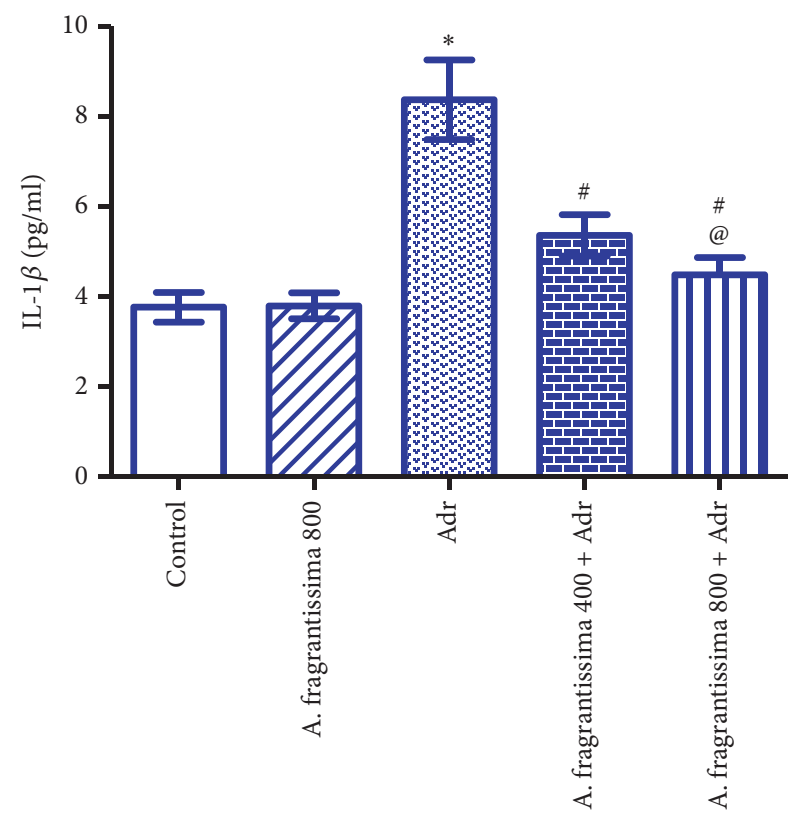

FIgURE 3: Effects of $A$. fragrantissima on serum concentration of IL- $1 \beta$ measured in control and Adr-induced cardiotoxic rats. Data are represented as mean \pm SE $(n=10)$. ${ }^{*}$ Significant versus control group, ${ }^{*}$ significant versus Adr group, and ${ }^{\circledR}$ significant between low and high dose group. $\mathrm{p} \leq 0.05$.

elevation. These results are consistent with many previous findings which suggested QT interval, an indicator of ventricular function to be prolonged during Adr toxicity via an oxidative stress mechanism $[29,32]$. In addition, 
TABLE 4: Effects of A. fragrantissima on heart tissue concentration of TBARS, GSH-PX, and GSH measured in control and Adr-induced cardiotoxic rats.

\begin{tabular}{lccc}
\hline Experimental Groups & $\begin{array}{c}\text { TBARS } \\
\text { (nmol/g tissue) }\end{array}$ & $\begin{array}{c}\text { GSH-PX } \\
\text { (nmol/mg tissue) }\end{array}$ & $\begin{array}{c}\text { GSH } \\
(\mathrm{nmol} / \mathrm{g} \text { tissue) }\end{array}$ \\
\hline Control & $4.76 \pm 0.49$ & $27.89 \pm 1.51$ & $4.34 \pm 0.08$ \\
A. fragrantissima 800 & $4.51 \pm 0.46$ & $28.12 \pm 1.87$ & $4.75 \pm 0.15$ \\
Adr & $9.16 \pm 0.84^{\mathrm{a}}$ & $12.73 \pm 1.66^{\mathrm{a}}$ & $1.78 \pm 0.06^{\mathrm{a}}$ \\
A. fragrantissima $400+\mathrm{Adr}$ & $6.29 \pm 0.53^{\mathrm{b}}$ & $23.61 \pm 1.58^{\mathrm{b}}$ & $3.61 \pm 0.12^{\mathrm{b}}$ \\
A. fragrantissima $800+\mathrm{Adr}$ & $5.18 \pm 0.44^{\mathrm{b}, \mathrm{c}}$ & $27.17 \pm 1.44^{\mathrm{b}, \mathrm{c}}$ & $4.14 \pm 0.09^{\mathrm{b}, \mathrm{c}}$ \\
\hline
\end{tabular}

Data are represented as mean $\pm \mathrm{SE}(\mathrm{n}=10)$. ${ }^{\mathrm{a}}$ Significant versus control group, ${ }^{\mathrm{b}}$ significant versus Adr group, and ${ }^{\mathrm{C}}$ significant between low and high dose group. $\mathrm{p} \leq 0.05$.

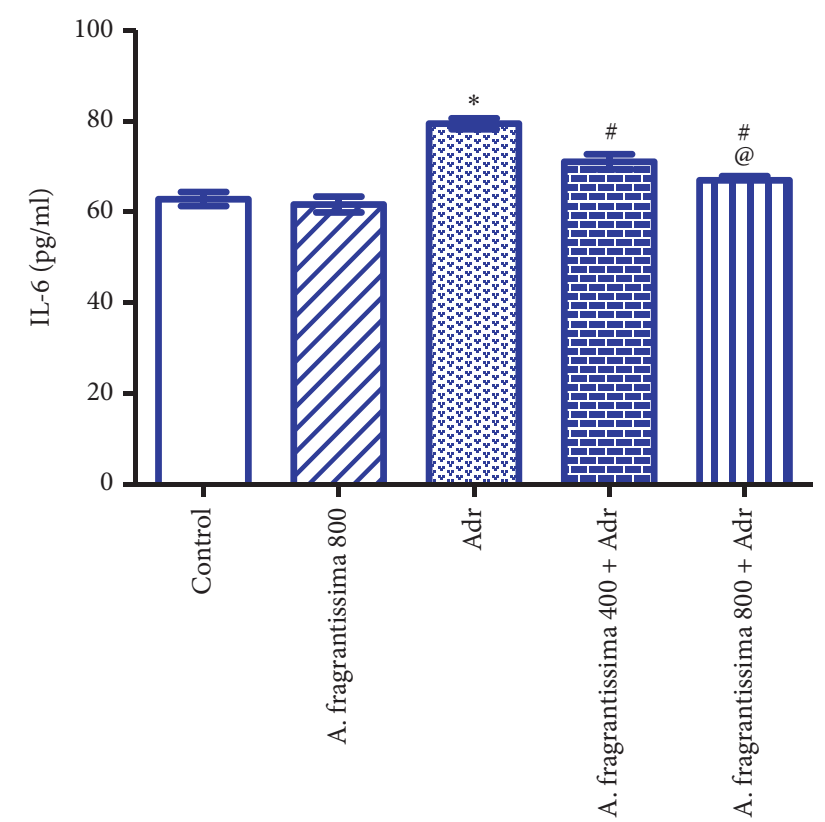

Figure 4: Effects of A. fragrantissima on serum concentration of IL-6 measured in control and Adr-induced cardiotoxic rats. Data are represented as mean $\pm \mathrm{SE}(\mathrm{n}=10)$. ${ }^{*}$ Significant versus control

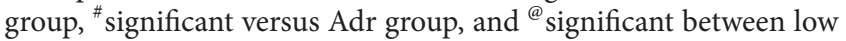
and high dose group. $\mathrm{p} \leq 0.05$.

Adr-increased ROS generation causes ST-segment elevation which is an important marker of ischemia [33]. Pretreatment of Adr injected rats with $A$. fragrantissima extract moderately ameliorated Adr-induced ST-segment elevation. However, $A$. fragrantissima extract failed to reverse Adr-induce tachycardia and QT prolongation.

The imbalance between free radicals and antioxidant enzymes is considered the most important mechanism behind Adr-developed cardiac toxicity $[30,34]$. The present results demonstrated that, following Adr injection, cardiac levels of TBARS were moderately increased, and that the levels of the cardiac antioxidant GSH-PX and GSH were moderately reduced. This information obviously points a case of distinct oxidative stress. These data are in consistent with the recent results of Benzer et al. [35]. A. fragrantissima extract moderately decreased the Adr-associated elevation of heart tissue content of TBARS, which is the product of Adrinduced lipid peroxidation. Furthermore, A. fragrantissima extract moderately increased the heart tissue content of GSHPX and GSH, which are important antioxidant defense of the heart. Similarly, a previous study has shown that Achilloid A, a sesquiterpene lactone, one of the most biologically active constituents of $A$. fragrantissima, could resist the oxidative stress caused by $\mathrm{H}_{2} \mathrm{O}_{2}$ in astrocytes via scavenging of ROS and blocking of $\mathrm{H}_{2} \mathrm{O}_{2}$-induced mitogen-activated protein kinase (MAPK) pathway [19]. This study also showed high levels of phenolic and flavonoid compounds in the A. fragrantissima extract. Like our results, the methanolic extract of $A$. fragrantissima had a large amount of phenolic and flavonoid compounds and exerted a strong antioxidant activity in the diphenylpicrylhydrazyl (DPPH) free radical scavenging assay $[20,36]$. The phenolic compounds display great ROS scavenging efficacy, via their reactivity as hydrogen or electron-donating substances, and metal ion chelating attributes [37, 38].

Several mechanistic pathways are probable to be implicated in Adr-induced cardiotoxicity including increased inflammatory reactions within the myocardium and promoted the release of proinflammatory cytokines including TNF- $\alpha$ and IL- $\beta$ [39]. In addition, novel clinical proof props the possible impact of IL-8 in atherosclerosis, both as a sign and as a probable medicinal goal. In the area of interventional cardiology, it was proposed that high serum concentration of IL-8 after percutaneous coronary intervention could prognosticate the evolution of cardiac failure in humans with acute myocardial infarction [40, 41]. NFkB signaling is a converging point for controlling downstream signaling cascades that include TNF- $\alpha$, IL-1 $\beta$, IL- 6 , IL-8, and transcription of other inflammatory genes [42].

Beyond their antioxidant effects, A. fragrantissima extract has frequently been shown to possess various anti-inflammatory and immunomodulatory effects [19]. The present study demonstrated that pretreatment with $A$. fragrantissima caused significant reductions in serum TNF$\alpha$, IL- $1 \beta$, and IL- 6 levels, suggesting a reliable cytoprotective action of $A$. fragrantissima against Adr-mediated release of proinflammatory mediators. Similarly, A. fragrantissima extract inhibited lipopolysaccharide (LPS)-induced 


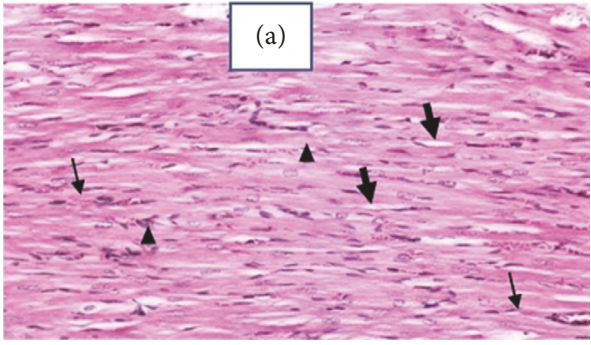

(a)

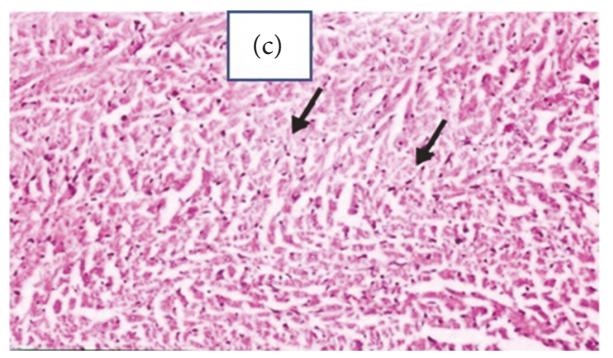

(c)

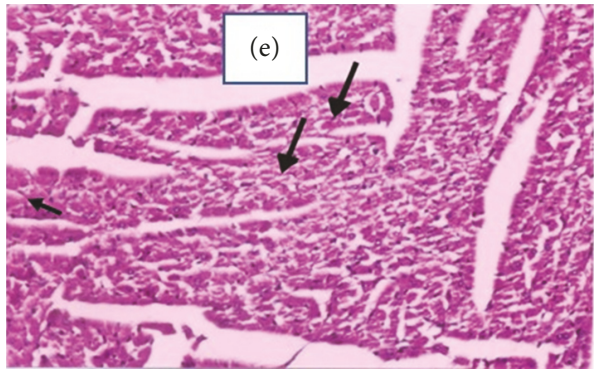

(e)

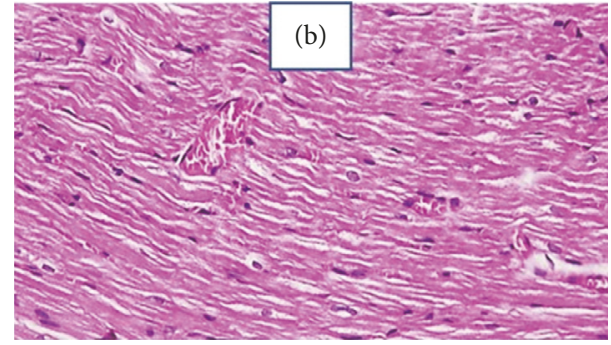

(b)

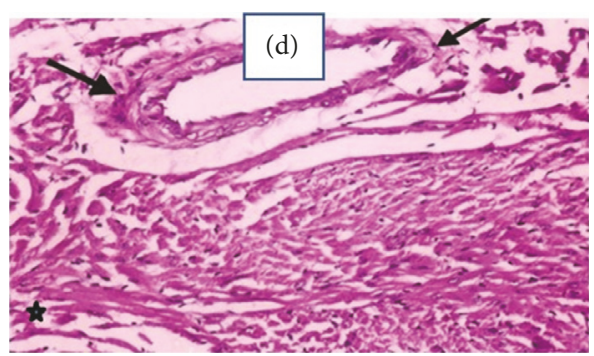

(d)

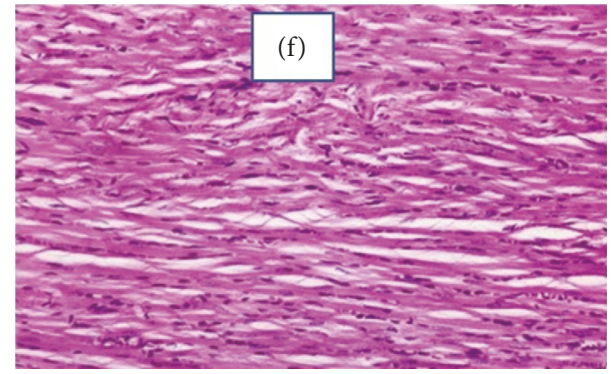

(f)

Figure 5: Effects of A. fragrantissima on the heart tissue histopathological changes detected by $\mathrm{H} \& \mathrm{E}$ staining in Adr-induced cardiotoxicity in rat (low power magnification $\times 200$ ). Photo (a) represents control group, cardiac muscle showing branching, anastomosing, and striated muscle fibers with central oval vesicular nuclei $(\bullet)$ and acidophilic cytoplasm. Notice the interstitial blood capillaries $(\longrightarrow)$ and spindle-shaped connective tissue cells (thick arrow). Photo (b) represents A. fragrantissima 800 group. Photo (c) represents Adr group, cardiac muscle showing pale cytoplasm with pyknotic nuclei (thick arrows). Focal areas of widely separated cardiac muscle fibers ranging from degenerated cytoplasm up to necrosis. Photo (d) represents Adr group, cardiac muscle showing the perivascular edema around a coronary blood vessel (thick arrows) together with marked disorganization of the thin degenerated fibers $(*)$. Notice the scattered pyknotic nuclei in the damaged fibers. Photo (e) represents A. fragrantissima $400+$ Adr group, showing necrosis of focal areas of degenerated cardiac muscle fiber (thick arrows). Notice the dark small pyknotic nuclei of muscle fibers with abnormal staining (arrow head). Photo (f) represents A. fragrantissima $800+$ Adr group, showing the control appearance of the branching, anastomosing, and striated cardiac muscle.

expression of TNF- $\alpha$ and IL-1 $\beta$ and downregulated ROS production from primary cultures of activated microglial cells [19]. The mechanism by which A. fragrantissima reduced TNF- $\alpha$, IL- $1 \beta$, and IL- 6 concentrations following Adr injection has not been elucidated in this work. However, the anti-inflammatory effect of $A$. fragrantissima extract may result from its antioxidant active constituents that can cross the cell membranes and scavenge the ROS intracellularly [19]. Therefore, the inhibitory effect of the A. fragrantissima extract on the expression of TNF- $\alpha$ and IL-1 $\beta$ might be attributed to inhibition of NFkB activation or to other signaling events leading to the production of proinflammatory molecules in myocytes such as protein kinase C or p38 MAPK [43].

\section{Conclusion}

The results of this study revealed that $A$. fragrantissima extract ameliorates Adr-induced cardiotoxicity as it ameliorated STsegment elevation and reduced $\mathrm{LDH}$ and CK-MB via an antioxidant (decreased TBARS and increased GSH and GSHPX) and anti-inflammatory (decreased TNF- $\alpha$, IL- $1 \beta$, and IL-6) mechanisms. Further studies are recommended to elucidate the specified active constituents of $A$. fragrantissima 


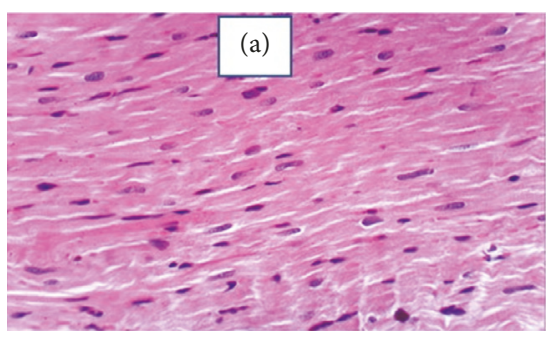

(a)

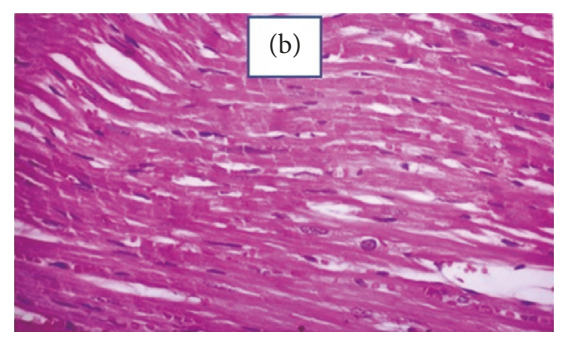

(b)

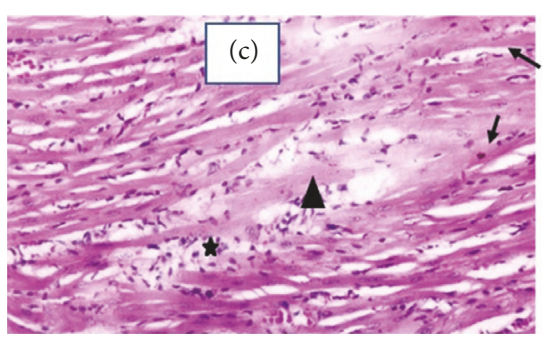

(c)

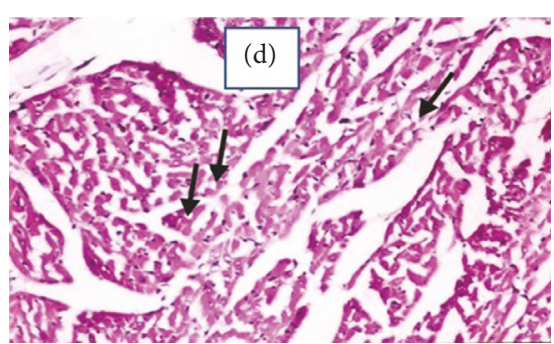

(d)

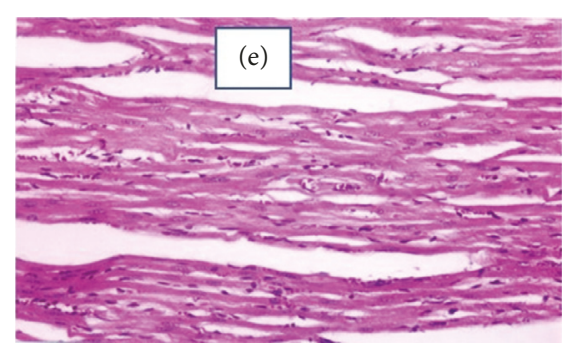

(e)

Figure 6: Effects of A. fragrantissima on the heart tissue histopathological changes detected by $\mathrm{H}$ \& $\mathrm{E}$ staining in control and Adr-induced cardiotoxicity in rat (high-power magnification $\times 400$ ). Photo (a) represents the cardiac muscle of control group. Photo (b) represents the cardiac muscle of $A$. fragrantissima 800 group, showing nearly normal cardiac muscle. Photo (c) represents the cardiac muscle of Adr group, showing focal disruption of some fibers $(\longrightarrow)$ with pale acidophilic sarcoplasm. Notice the marked focal aggregation of inflammatory mononuclear cells $(*)$, interstitial fibroblasts (arrow head), and edema in between the fibers. Photo (d) represents the cardiac muscle of $A$. fragrantissima 400 + Adr group, showing the widely separated degenerated myocardial fibers with pale homogenous cytoplasm (thick arrows). Notice focal cytoplasmic vacuolization of some cardiac muscle fibers $(\longrightarrow)$. Photo (e) represents the cardiac muscle of A. fragrantissima 800 + Adr group, showing the normal branched appearance of the cardiac muscle fibers.

which are lies beneath its cardioprotective effect through the antioxidant and anti-inflammatory actions.

\section{Data Availability}

The data and materials supporting the conclusions of this article are included within the article.

\section{Conflicts of Interest}

The authors declare that they have no conflicts of interest to declare.

\section{References}

[1] M. Fernandez-Chas, M. J. Curtis, and S. A. Niederer, "Mechanism of doxorubicin cardiotoxicity evaluated by integrating multiple molecular effects into a biophysical model," British Journal of Pharmacology, vol. 175, no. 5, pp. 763-781, 2018.

[2] D. Mele, C. G. Tocchetti, P. Pagliaro et al., "Pathophysiology of anthracycline cardiotoxicity," Journal of Cardiovascular Medicine, vol. 17, pp. e3-ell, 2016.

[3] N. Maurea, C. Coppola, G. Piscopo et al., "Pathophysiology of cardiotoxicity from target therapy and angiogenesis inhibitors," Journal of Cardiovascular Medicine, vol. 17, pp. e19-e26, 2016.

[4] T. Šimůnek, M. Štěrba, O. Popelová, M. Adamcová, R. Hrdina, and V. Gerši, "Anthracycline-induced cardiotoxicity: overview of studies examining the roles of oxidative stress and free cellular iron," Pharmacological Reports, vol. 61, no. 1, pp. 154171, 2009.

[5] K. Wu, S. J. Schwartz, E. A. Platz et al., "Variations in plasma lycopene and specific isomers over time in a cohort of U.S. men," Journal of Nutrition, vol. 133, no. 6, pp. 1930-1936, 2003.

[6] T. A. A. El-Aziz, R. H. Mohamed, H. F. Pasha, and H. R. Abdel-Aziz, "Catechin protects against oxidative stress and inflammatory-mediated cardiotoxicity in adriamycin-treated rats," Clinical and Experimental Medicine, vol. 12, no. 4, pp. 233240, 2012.

[7] R. Guo, K. Wu, J. Chen et al., "Exogenous hydrogen sulfide protects against doxorubicin-induced inflammation and cytotoxicity by inhibiting p38MAPK/NF $\kappa$ B pathway in $\mathrm{H} 9 \mathrm{c} 2$ cardiac cells," Cellular Physiology and Biochemistry, vol. 32, no. 6, pp. 1668-1680, 2013.

[8] J. Zhu, J. Zhang, L. Zhang et al., "Interleukin-1 signaling mediates acute doxorubicin-induced cardiotoxicity," Biomedicine \& Pharmacotherapy, vol. 65, no. 7, pp. 481-485, 2011.

[9] A. Hosseini, E. Bakhtiari, and S. H. Mousavi, "Protective effect of hibiscus sabdariffa on doxorubicin-induced cytotoxicity in H9c2 cardiomyoblast cells," Iranian Journal of Pharmaceutical Research, vol. 16, no. 2, pp. 708-713, 2017.

[10] I. Capasso, E. Esposito, N. Maurea et al., "Combination of inositol and alpha lipoic acid in metabolic syndrome-affected women: a randomized placebo-controlled trial," Trials, vol. 14, no. 1, p. 273, 2013.

[11] P. Møller, S. Loft, C. Lundby, and N. V. Olsen, "Acute hypoxia and hypoxic exercise induce DNA strand breaks and oxidative DNA damage in humans," The FASEB Journal, vol. 15, no. 7, pp. 1181-1186, 2001. 
[12] K. Nakamura, K. Fushimi, H. Kouchi et al., "Inhibitory effects of antioxidants on neonatal rat cardiac myocyte hypertrophy induced by tumor necrosis factor-alpha and angiotensin II," Circulation, vol. 98, no. 8, pp. 794-799, 1998.

[13] M. M. Shabana, Y. W. Mirhom, A. A. Genenah, E. A. Aboutabl, and H. A. Amer, "Study into wild Egyptian plants of potential medicinal activity. Ninth communication: hypoglycaemic activity of some selected plants in normal fasting and alloxanised rats," Archiv für Experimentelle Veterinärmedizin, vol. 44, no. 3, pp. 389-394, 1990.

[14] E. H. Mustafa, M. Abu Zarga, and S. Abdalla, "Effects of cirsiliol, a flavone isolated from Achillea fragrantissima, on rat isolated ileum," General Pharmacology: The Vascular System, vol. 23, no. 3, pp. 555-560, 1992.

[15] Z. Yaniv, A. Dafni, J. Friedman, and D. Palevitch, "Plants used for the treatment of diabetes in Israel," Journal of Ethnopharmacology, vol. 19, no. 2, pp. 145-151, 1987.

[16] I. I. Hamdan and F. U. Afifi, "Studies on the in vitro and in vivo hypoglycemic activities of some medicinal plants used in treatment of diabetes in Jordanian traditional medicine," Journal of Ethnopharmacology, vol. 93, no. 1, pp. 117-121, 2004.

[17] S. M. Ezzat and M. M. Salama, "A new $\alpha$-glucosidase inhibitor from Achillea fragrantissima (Forssk.) Sch. Bip. growing in Egypt," Natural Product Research (Formerly Natural Product Letters), vol. 28, no. 11, pp. 812-818, 2014.

[18] A. Elmann, A. Telerman, S. Mordechay et al., "Downregulation of microglial activation by achillolide A," Planta Medica, vol. 81, no. 3, pp. 215-221, 2015.

[19] A. A. Mohamed, S. I. Ali, F. K. El-Baz, and W. M. El-Senousy, "New insights into antioxidant and antiviral activities of two wild medicinal plants: achillea fragrantissima and Nitraria Retusa," International Journal of Pharma and Bio Sciences, vol. 6, no. 1, pp. P708-P722, 2015.

[20] I. Merfort, "Perspectives on sesquiterpene lactones in inflammation and cancer," Current Drug Targets, vol. 12, no. 11, pp. 1560-1573, 2011.

[21] S.-K. Kim, S.-B. Cho, and H.-I. Moon, "Neuroprotective effects of a sesquiterpene lactone and flavanones from Paulownia tomentosa Steud. against glutamate-induced neurotoxicity in primary cultured rat cortical cells," Phytotherapy Research, vol. 24, no. 12, pp. 1898-1900, 2010.

[22] E. M. Choi, G.-H. Kim, and Y. S. Lee, "Protective effects of dehydrocostus lactone against hydrogen peroxide-induced dysfunction and oxidative stress in osteoblastic MC3T3-E1 cells," Toxicology in Vitro, vol. 23, no. 5, pp. 862-867, 2009.

[23] K. Gach, A. Długosz, and A. Janecka, "The role of oxidative stress in anticancer activity of sesquiterpene lactones," NaunynSchmiedeberg's Archives of Pharmacology, vol. 388, no. 5, pp. 477-486, 2015.

[24] A. M. Alenad, N. A. Al-Jaber, S. Krishnaswamy, S. M. Yakout, M. N. Al-Daghri, and M. S. Alokail, "Achillea fragrantissima extract exerts its anticancer effect via induction of differentiation, cell cycle arrest and apoptosis in chronic myeloid leukemia (CML) cell line K562," Journal of Medicinal Plants Research, vol. 7, no. 21, pp. 1561-1567, 2013.

[25] A. J. Harborne, Phytochemical Methods: A Guide to Modern Techniques of Plant Analysis, Chapman and Hall, Springer, Netherlands, 3rd ed edition, 1998.

[26] N. A. El-Shitany, S. El-Haggar, and K. El-desoky, "Silymarin prevents adriamycin-induced cardiotoxicity and nephrotoxicity in rats," Food and Chemical Toxicology, vol. 46, no. 7, pp. 24222428, 2008.
[27] M. Hosseini, F. Harandizadeh, S. Niazamand, M. Soukhtanloo, and M. Mahmoudabady, "Antioxidant effect of Achillea wilhelmsii extract on pentylenetetrazole (seizure model)-induced oxidative brain damage in Wistar rats," Indian Journal of Physiology and Pharmacology, vol. 57, no. 4, pp. 418-424, 2013.

[28] A. Azhar and H. M. El-Bassossy, "Pentoxifylline alleviates cardiac ischemia and dysfunction following experimental angina in insulin resistance," PLoS ONE, vol. 9, no. 5, article e98281, 2014.

[29] H. A. H. Khattab, N. A. El-Shitany, and A. K. S. Al-Lily, "Effect of roselle (Hibiscus sabdariffa) against adriamycin inducedcardiotoxicity in male rats," The International Journal of Pharmaceutical Research and Allied Sciences, vol. 5, no. 3, pp. 519534, 2016.

[30] M. C. Asensio-López, F. Soler, D. Pascual-Figal, F. FernándezBelda, and A. Lax, "Doxorubicin-induced oxidative stress: the protective effect of nicorandil on HL-1 cardiomyocytes," PLoS ONE, vol. 12, no. 2, article e0172803, 2017.

[31] D. Jain, T. Ahmad, M. Cairo, and W. Aronow, "Cardiotoxicity of cancer chemotherapy: identification, prevention and treatment," Annals of Translational Medicine, vol. 5, no. 17, pp. 348348, 2017.

[32] B. C. Koti, S. Nagathan, A. Vishwanathswamy, P. C. Gadad, and A. Thippeswamy, "Cardioprotective effect of Vedic Guard against doxorubicin-induced cardiotoxicity in rats: a biochemical, electrocardiographic, and histopathological study," Pharmacognosy Magazine, vol. 9, no. 34, pp. 176-181, 2013.

[33] E. M. El-Sayed, A. S. Abd El-azeem, A. A. Afify, M. H. Shabana, and H. H. Ahmed, "Cardioprotective effects of Curcuma longa L. extracts against doxorubicin-induced cardiotoxicity in rats," Journal of Medicinal Plants Research, vol. 5, no. 17, pp. 40494058, 2011.

[34] S. T. Duggan and G. M. Keating, "Pegylated Liposomal doxorubicin," Drugs, vol. 71, no. 18, pp. 2531-2558, 2011.

[35] F. Benzer, F. M. Kandemir, M. Ozkaraca, S. Kucukler, and C. Caglayan, "Curcumin ameliorates doxorubicin-induced cardiotoxicity by abrogation of inflammation, apoptosis, oxidative DNA damage, and protein oxidation in rats," Journal of Biochemical and Molecular Toxicology, vol. 32, no. 2, p. e22030, 2018.

[36] A. A. Shahat and M. S. Alsaid, "Antioxidant capacity and polyphenolic content of seven Saudi Arabian medicinal herbs traditionally used in Saudi Arabia," Indian Journal of Traditional Knowledge, vol. 14, no. 1, pp. 28-35, 2015.

[37] C. A. Rice-Evans, N. J. Miller, and G. Paganga, "Structureantioxidant activity relationships of flavonoids and phenolic acids," Free Radical Biology \& Medicine, vol. 20, no. 7, pp. 933956, 1996.

[38] Y. Lu and L. Y. Foo, "Antioxidant activities of polyphenols from sage (Salvia officinalis)," Food Chemistry, vol. 75, no. 2, pp. 197202, 2001.

[39] R. A. Shaker, S. H. Abboud, H. C. Assad, and N. Hadi, "Enoxaparin attenuates doxorubicin induced cardiotoxicity in rats via interfering with oxidative stress, inflammation and apoptosis," BMC Pharmacology \& Toxicology, vol. 19, no. 1, p. 3, 2018.

[40] V. Quagliariello, R. Vecchione, C. Coppola et al., "Cardioprotective effects of nanoemulsions loaded with antiinflammatory nutraceuticals against doxorubicin-induced cardiotoxicity," Nutrients, vol. 10, no. 9, 2018, pii. E1304. 
[41] S. Apostolakis, K. Vogiatzi, V. Amanatidou, and D. A. Spandidos, "Interleukin 8 and cardiovascular disease," Cardiovascular Research, vol. 84, no. 3, pp. 353-360, 2009.

[42] D. Vyas, G. Laput, and A. K. Vyas, "Chemotherapy-enhanced inflammation may lead to the failure of therapy and metastasis," OncoTargets and Therapy, vol. 7, pp. 1015-1023, 2014.

[43] A. Elmann, S. Mordechay, H. Erlank, A. Telerman, M. Rindner, and R. Ofir, "Anti-Neuroinflammatory effects of the extract of Achillea fragrantissima," BMC Complementary and Alternative Medicine, vol. 11, p. 98, 2011. 

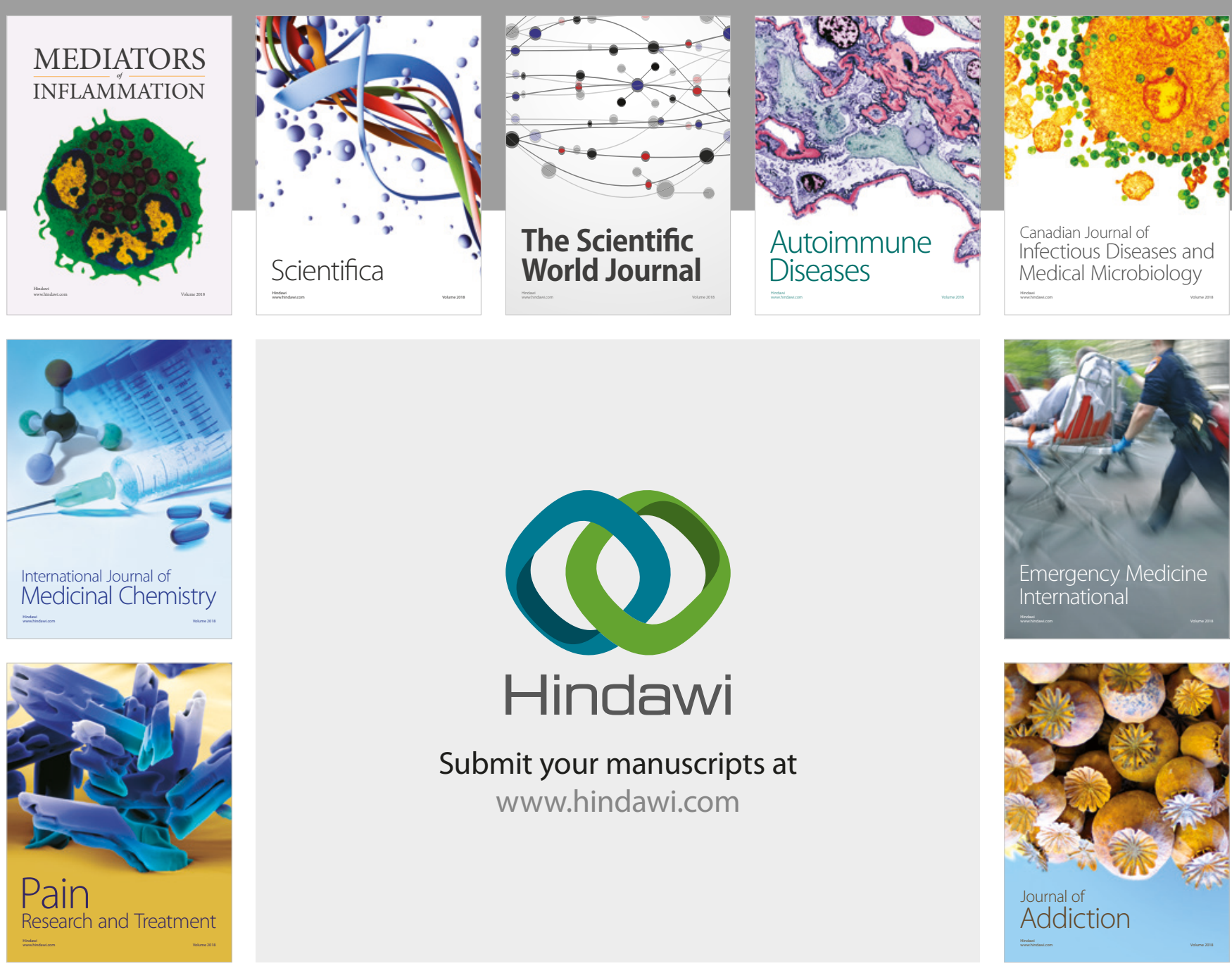

Canadian Journal of
Infectious Diseases and Medical Microbiology

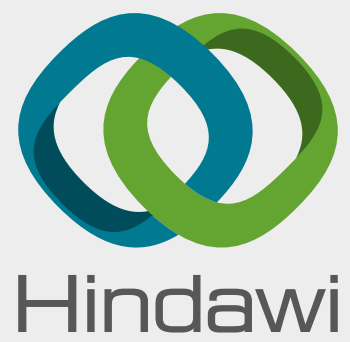

Submit your manuscripts at

www.hindawi.com
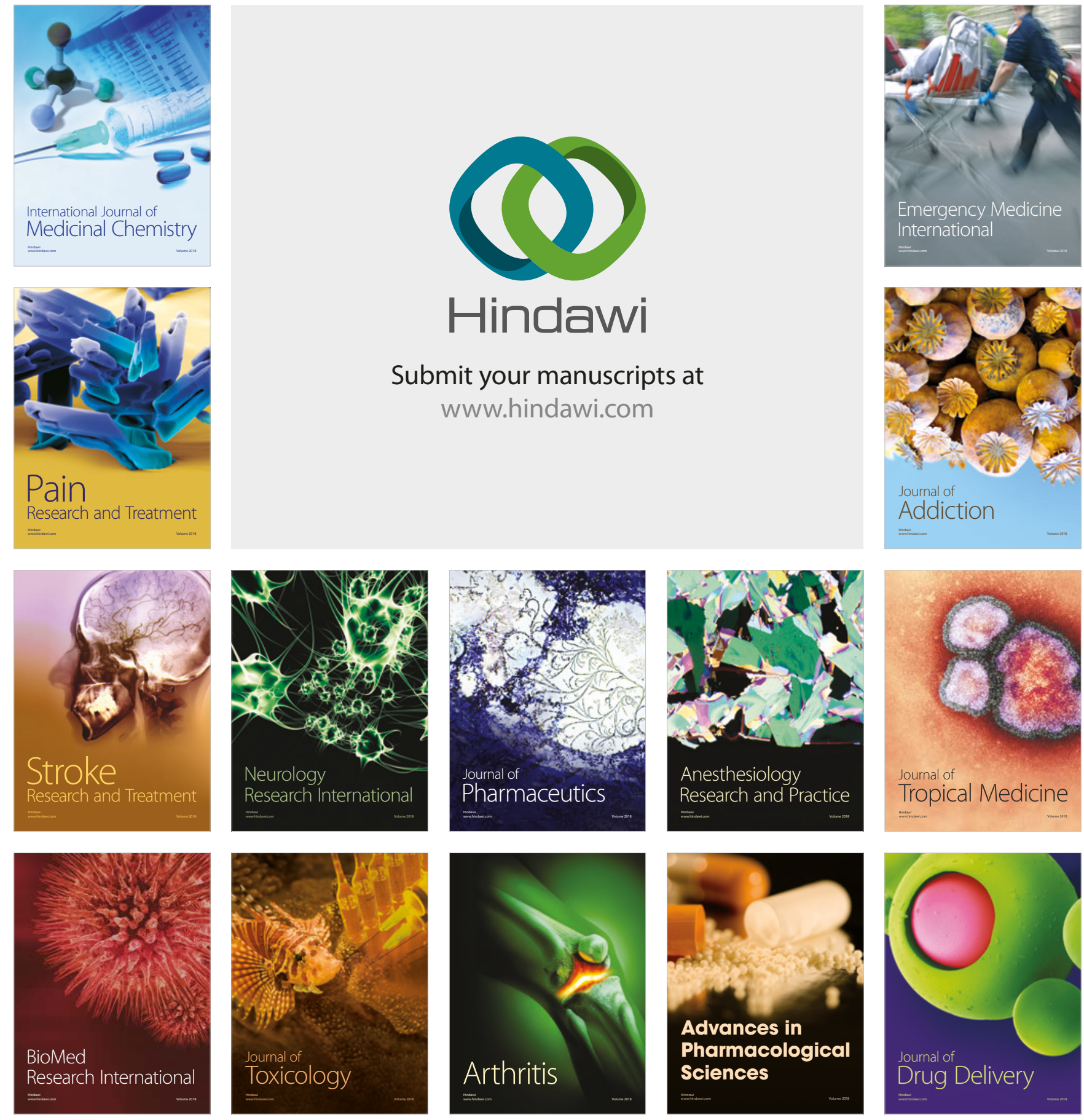